\title{
Electro-optical modulation of sub-terahertz radiation with superconducting metamaterial
}

\author{
V. Savinov ${ }^{1, *}$, V. A. Fedotov ${ }^{1}$, P. A. J. de Groot ${ }^{2}$ and N. I. Zheludev ${ }^{1}$ \\ ${ }^{1}$ Optoelectronics Research Centre and Centre for Photonic Metamaterials, University of Southampton, \\ Southampton SO17 1BJ, United Kingdom \\ ${ }^{2}$ School of Physics and Astronomy and Centre for Photonic Metamaterials, University of Southampton, \\ Southampton SO17 1BJ, United Kingdom \\ *vs1106@orc.soton.ac.uk
}

\begin{abstract}
We present the first demonstration of electro-optical modulator of sub-terahertz radiation where intensity and phase modulation is achieved by running current through a superconducting network of niobium meta-molecules supporting a high-Q resonance. OCIS codes: (160.3918) Metamaterials; (230.2090) Electro-optical device; (250.4110) Modulators
\end{abstract}

We report on the first experimental realization of the electrical control in superconducting metamaterials, which enables an efficient and fast modulation of free-space sub-terahertz radiation. The modulation is underpinned by controlling the superconductive state of the niobium framework with current. Only few tens of volts of controlling signal are sufficient for 50\% sub-terahertz intensity modulation and modulation rates up to several hundreds of $\mathrm{kHz}$ have been observed.

a)

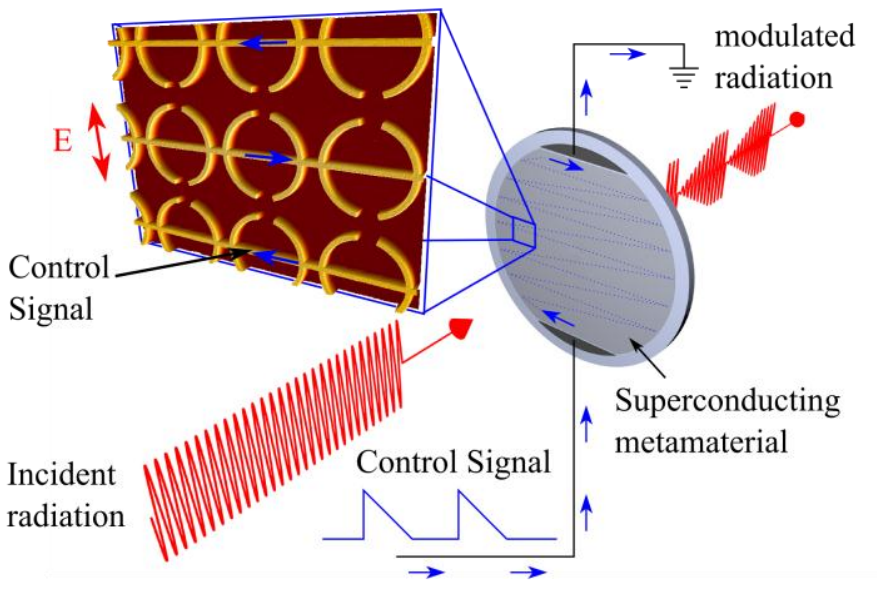

b)

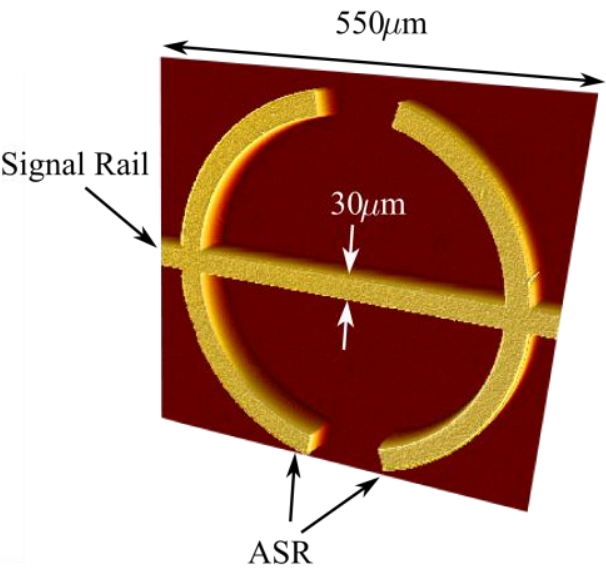

\begin{abstract}
Fig. 1: The electro-optical modulator based on superconducting metamaterial. a) Schematic of the modulator. The zoom-in shows an profile microscope image (colour represents height) of the metamaterial array, with blue arrows indicating the direction of the control current and and red arrow showing the polarization of the incident radiation. b) A close-up view of the unit cell of the metamaterial.
\end{abstract}

The metamaterial modulator (see Fig. 1) was fabricated by patterning a thin niobium film deposited on a sapphire substrate. The patterning produced a planar array of asymmetrically split rings intersected by a straight niobium wire - the signal rail that connects all meta-atoms in the array into a single chain. The metamaterial was placed inside a closed-cycle optical cryostat. Metamaterial modulator was characterized in the spectral range 75 $-110 \mathrm{GHz}$ using mm-wave horn antennas and network analyser (Fig. 2).

Fig. 3 shows the transmission of the metamaterial for temperatures above and below the superconducting transition in niobium. In normal state $(\theta=100 \mathrm{~K})$ niobium is a poor conductor and the resonant response of the metamaterial is suppressed. In the superconducting state $(\theta=4 \mathrm{~K})$ the dissipative losses in niobium are significantly reduced giving rise to a sharp collective sub-radiant (trapped-mode) resonance associated with the asymmetry of the split ring resonators. The resonance is located at the frequency of $99.5 \mathrm{GHz}$ and has a high quality factor $\mathrm{Q} \sim 130$.

We demonstrated electro-optical control of transmitted sub-terahertz radiation by applying triangle ramp pulses (from a voltage source) to the metamaterial niobium framework and measuring metamaterial's transmission at frequencies across the resonance. The current passing through the niobium metamaterial 
framework affects its superconducting state and in turn drastically increases Ohmic losses in the arcs of the split rings modifying their electromagnetic response.

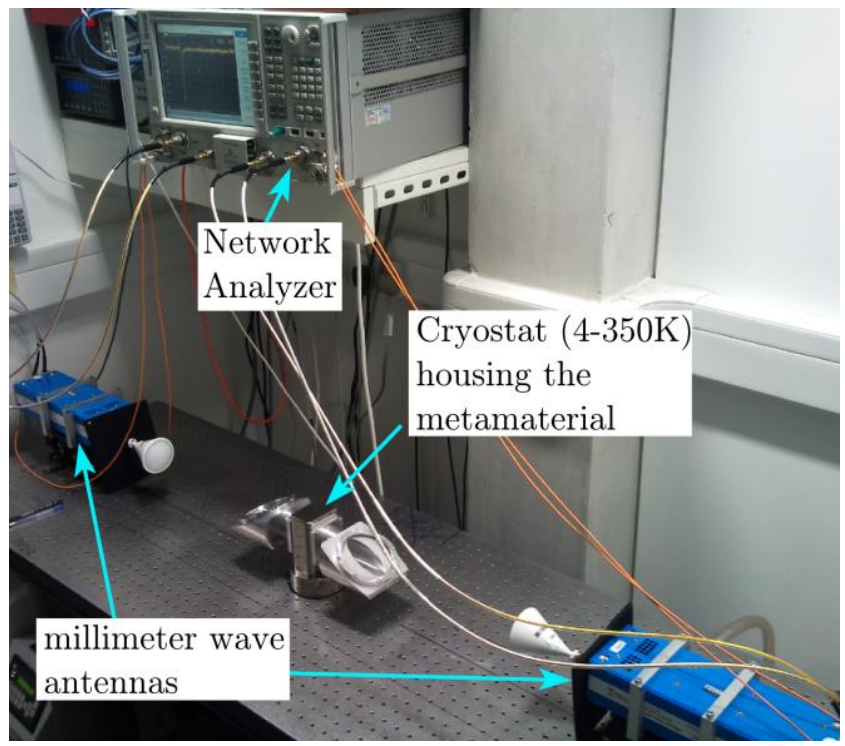

Fig. 2: The experimental setup for characterization of metamaterial showing network analyser, millimetre wave horn antennas and a closedcycle helium-cooled optical cryostat.

The change in transmission as a function of time is shown on the inset in Fig. 3, where different curves correspond to different maximum values of the applied voltage ramp. Transmission modulation up to $50 \%$ has been observed. The change in transmission follows the applied ramp closely while the voltage is increased, but as soon as the ramp pulse is over the transmission returns to original level following an exponential-like decay, with decay time of $\sim 22 \mu \mathrm{s}$. The decay time can be reduced substantially for small amplitude modulation (not shown here) where modulation rates of several hundreds of $\mathrm{kHz}$ have been observed. Modulation of transmission has also been observed with trains of electromagnetic pulses and continuous AC modulation up to instrumental frequency limit of $100 \mathrm{kHz}$.

We discuss various microscopic mechanisms behind of the modulation effect and their dynamics.

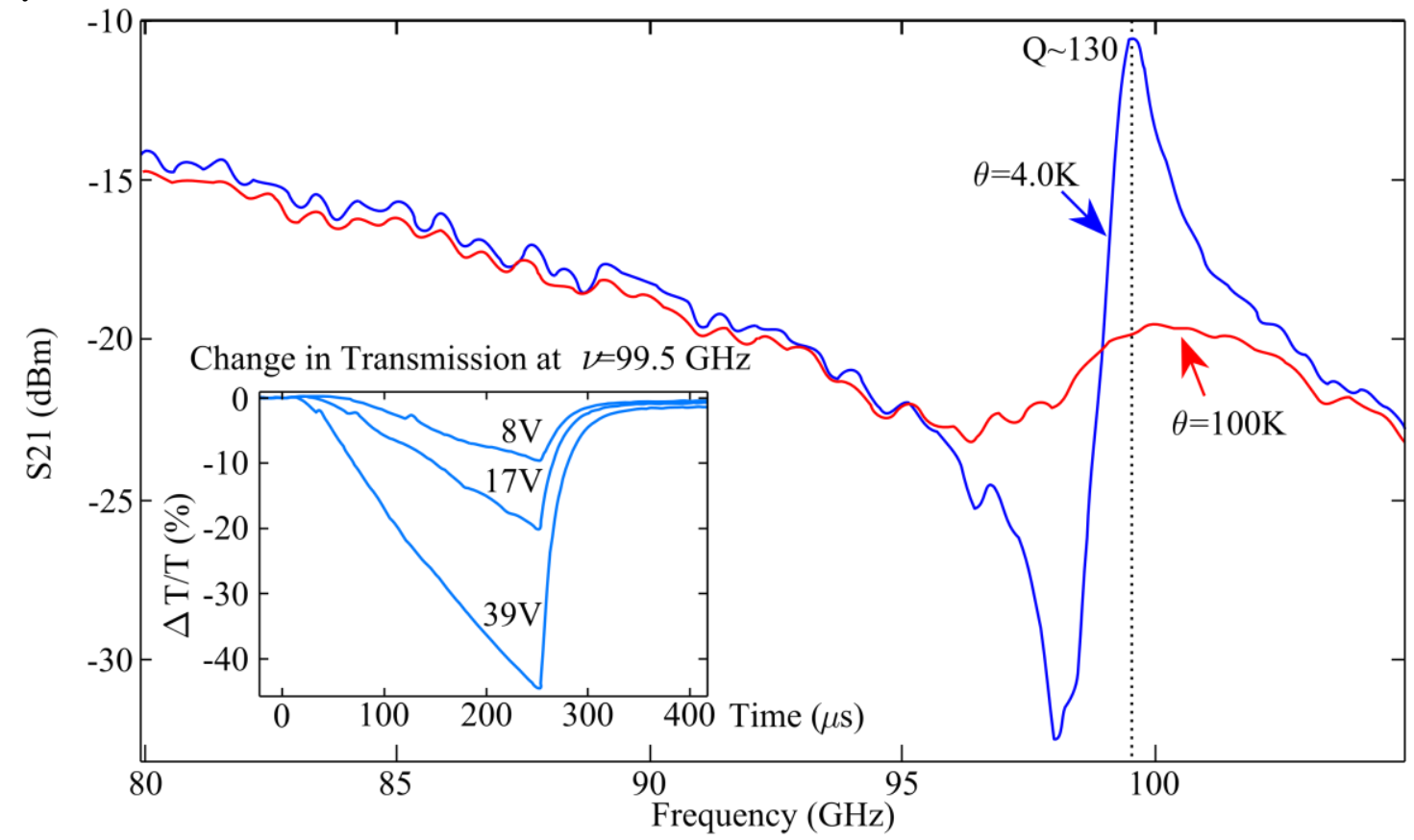

Fig. 3: Transmission and switching characteristics of sub-terahertz modulator based on niobium metamaterial. Main plot shows transmission spectra of the metamaterial in normal and superconducting states. At $4 \mathrm{~K}$ the metamaterial shows an extremely sharp resonance at $v=99.5$ $\mathrm{GHz}$ with a quality factor of $\mathrm{Q} \sim 130$. Inset shows changes in transmission level of the metamaterial at $v=99.5 \mathrm{GHz}$ in response to applied voltage ramps. The duration of the ramps is $250 \mu \mathrm{s}$. Different curves correspond to different peak amplitudes of the ramps. 